\title{
MicroRNAs Expression Profiles in Platelet-Derived Exosomes From Coronary In-Stent Restenosis and the Potential Markers
}

\author{
Lingdi Hua \\ Southeast University \\ Yong Qiao \\ Department of Cardiology, Zhongda Hospital affiliated to Southeast University \\ Linqing Li \\ Southeast University \\ Yuhan Qin \\ Southeast University \\ Dong Wang \\ Department of Cardiology, Zhongda Hospital affiliated to Southeast University \\ Gaoliang Yan \\ Department of Cardiology, Zhongda Hospital affiliated to Southeast University \\ Chengchun Tang ( $\nabla$ tangchengchun@hotmail.com) \\ Department of Cardiology, Zhongda Hospital affiliated to Southeast University
}

\section{Research Article}

Keywords: In-stent restenosis (ISR), Platelet-derived Exosomes(PDEs), MicroRNA, qRT-PCR

Posted Date: January 12th, 2021

DOI: https://doi.org/10.21203/rs.3.rs-129758/v1

License: (c) (i) This work is licensed under a Creative Commons Attribution 4.0 International License. Read Full License 


\section{Abstract}

Background: We conducted this study was to identify any difference in platelet-derived exosome micro-RNA (microRNA) profile between patients with in-stent restenosis and patients without restenosis using gene sequencing.

Method: Platelet-derived exosomes(PDEs) were isolated through ultracentrifugation and validated by Western blotting, transmission electron microscope and NanoSight analysis. The platelet-derived exosome microRNA expression profile was identified by Illumina Hiseq2000/2500 sequencing, and validated by qRT-PCR. Finally, the potential functions of microRNAs were predicted using miRNA-gene-GO network and miRNA-gene-KEGG network.

Result: We identified several differentially expressed platelet-derived exosome microRNAs. In particular, we found that the microRNA PC-3p-75179_24 was up-regulated, while hsa-miR-133a-3p_R+1 and hsa-miR-589-5p_R-1 were down-regulated in patients with in-stent restenosis which were further validated by qRT-PCR results. Our results also showed their potential miRNA-gene-GO and miRNA-gene-KEGG interaction.

Conclusion: In summary, our study reveals for the first time microRNA expression is altered, in platelet-derived exosomes in patients with in-stent restenosis, with specific up-regulation of microRNA PC-3p-75179_24 and down-regulation of hsa-miR133a-3p_R+1 and hsa-miR-589-5p_R-1. Our findings supports the need to explore the potential of these microRNAs as biomarkers and the pathological effects on in-stent restenosis.

\section{Background}

Percutaneous coronary intervention $(\mathrm{PCl})$ has become the main choice for treatment of acute coronary syndrome. However, patients treated with PCl tend to have a greater risk of restenosis[1]. In-stent restenosis (ISR) is defined as $5 \mathrm{~mm}$ proximal or distal segments in or near the stent that reappear $>50 \%$ lumen stenosis during follow-up angiography. Despite the widespread use of drug-eluting stents and improvements in drug development, ISR remains a significant prognostic factor for percutaneous vascular revascularization[2]. The pathophysiological process of ISR is complicated, and includes three stages: injured vascular remodeling, inflammatory reaction and proliferation and migration of smooth muscle cells. Vascular endothelial injury and inflammation are the initial factors of ISR. Proliferation and migration of vascular smooth muscle cells and excessive thickening of the vascular neointima are the central factors leading to ISR[3]. In addition, emerging evidence indicates that low-grade chronic inflammation is a primary mechanism that underlies ISR, as it promotes vascular smooth muscle cells (VSMCs) to proliferate and migrate to the intima, causing neointimal hyperplasia[4, 5]. Platelets are thought to have important functions in promoting restenosis[6]. During balloon angioplasty or stent implantation, once the integrity of the endothelium has been destroyed, exposure to the endothelium leads to extensive activation, adhesion and aggregation of platelets within lesions. Next, platelets secrete prothrombin and additional inflammatory factors that together promote neointimal hyperplasia[7]. The exosome, type of vesicle, has a diameter of 30-150 nm and a cup-shaped lipid bilayer membrane[8, 9]. As exosomes carry functional proteins, RNA and lipids, they are thought to be messengers for intercellular communication and have become diagnostic markers for many different diseases[10-12]. Exosomes carry out the key function of intercellular communication by transferring bioactive substances (including proteins and microRNAs,) to receptor cells or activating signal transduction pathways in target cells[13].

MicroRNAs (miRNAs) are an endogenous non-coding small RNA, composed of 21-22 nucleotides[14]. Many studies have indicated that miRNAs are involved in a variety of different cellular processes, including differentiation, proliferation, metastasis and apoptosis[15]. The main function of miRNAs is the regulation of gene expression at the post-transcriptional level though degradation of target mRNAs and / or inhibition of mRNA translation[16]. As an important negative regulator of gene expression, thousands of miRNAs play important physiological and pathophysiological roles in humans[17, 18]. With regards to PDEs, studies have shown that miRNA-223 and miRNA-339 induce platelet aggregation and regulate the coagulation pathway in atherosclerosis[19]. Additionally, miRNA-21 and miRNA-223 have been demonstrated to up-regulate TNF- $a$ - induced ICAM-1 expression in human umbilical vein endothelial cells via a MAPK and NF-k $\beta$ dependent manner[20]. 
Furthermore, miRNA-320 inhibits, and miRNA-191 induces the expression of ICAM-1 in endothelial cells that are involved in inflammation[21, 22].

Upon studying the mechanism of coronary artery ISR, studies have validated that platelet activation can increase ISR incidence[23, 24]. However, the relationship between PDEs and the mechanism in coronary artery ISR has not yet been elucidated.

\section{Methods}

\section{Study subjects (patients and samples)}

Venous blood samples were acquired from 26 patients that underwent coronary stenting at the Department of Cardiology, Zhongda Hospital affiliated to the Medical College of Southeast University between the year of 2019 to 2020. All participants provided written consent prior to participating in the study.Study approval was obtained from the Human Subjects Committee at the Zhongda Hospital, Southeast University. Subjects were enrolled in the study according to the following inclusion criteria: 1) between the ages of $45-75 ; 2$ ) after stent implantation, the patients adhere to completion of the secondary prevention of coronary heart disease; 3 )the type of stent placed within the patient stent is common, and the surgery was conducted in the last 1 to 3 years. The exclusion criteria included diagnosis of diabetes mellitus, acute and chronic infection, hepatic and renal insufficiency, hematological diseases, severe bleeding, autoimmune diseases, malignant tumors, severe trauma, nonatherosclerotic acute myocardial infarction. The patients' plasma samples were collected in EDTA tubes. The patients were divided into either restenosis group or the no restenosis group (control group), according to their coronary angiography results. The blood was centrifuged at $4^{\circ}, 2000 \mathrm{rpm}$ centrifugation for $15 \mathrm{~min}$ within the first hour of collection. The plasma samples were stored at- $80^{\circ} \mathrm{C}$ until further processing. Three patients with restenosis and three patients without restenosis were randomly selected for genetic sequencing, while the remain samples were validated using by qRT-PCR.

\section{Isolation and purification of exosomes}

We used the TEN solution(0. $05 \mathrm{~mol} / \mathrm{L}$ Tris- $\mathrm{HCl}, 0.15 \mathrm{~mol} / \mathrm{L} \mathrm{NaCl}, 6 \mathrm{mmol} / \mathrm{L}$ EDTA-2Na,pH7. 4) to suspend platelet. Next, we extracted the platelet exosomes, which were then isolated and purified using the Platelet Exosome Isolation Kit (Invitrogen), as per the manufacturer's direction. We obtained the platelet exosome as a precipitate, which was then re-suspended in 50 to 200 $\mu$ I PBS and stored at $-80^{\circ} \mathrm{C}$.

\section{Transmission electron microscopy (TEM), Nanoparticle Tracking Analysis (NTA) and Western blotting(WB)}

The exosomes were fixed using glutaraldehyde for two hours. Next, we added 5-10 $\mu$ I of exosome solution to copper mesh, and let it adsorb for appropximately $10 \mathrm{~min}$ at room temperature. We carefully absorbed the excess liquid with filter paper. Then, $10 \mu$ I of $2 \%$ phosphotungstic acid solution $(\mathrm{pH}=6.5)$ was added to the copper net. Exosome staining was conducted by treating for $2 \mathrm{~min}$ at room temperature. Then, we carefully absorbed the excess dye with filter paper and dried the copper net at room temperature. The exosomes were observed on the machine, at a voltage of $120 \mathrm{kV}$. The particle size, concentrations and distribution of exosomes were analyzed using Nanoparticle Tracking Analysis (NTA) by NanoSight NS300 (Malvern Instruments, Shanghai, China).

Exosome protein denaturation was carried out as follows. After exosome lysate centrifugation, the supernatant was collected and $5 \times$ SDS-PAGE protein up-sample buffer was added according to 4:1 ratio. The EP tube was heated in a metal bath at $100^{\circ} \mathrm{C}$ for 10 minutes in order to denature the protein. After cooling, the protein was stored at $-80^{\circ} \mathrm{C}$ for future use. The BCA protein detection kit (DHbiotech, Shanghai) was utilized to detect protein concentration according to the manufacturer's instructions. The expression of various exosome markers (CD9, CD69 and TSG101) was analyzed by Western blotting. Next, proteins were transferred onto a polyvinylidene difluoride(PVDF) membrane. We used a rabbit monoclonal antibody to CD9 and CD69 (Abcam) and mouse monoclonal antibody to TSG101 (Abcam) with dilution ratio of 1:1000 and secondary antibody (Abcam) at 1:10 000 dilution. 


\section{Extraction of total RNA, library Construction and RNA sequencing analysis}

Using RNAprep pure cell Kit (TianGen BioTech, China), we isolated total RNA from PDEs. The RNA concentrations was validated by a NanoDrop ND-1000 spectrophotometer (Thermo Scientific). The experimental process was conducted according to instructions provided by lllumina, including the preparation of library and sequencing experiments. The small RNA sequencing library was prepared by TruSeq Small RNA Sample Prep Kits (Illumina, San Diego, USA). After the library was prepared, it was sequenced by Illumina Hiseq2000/2500, with a sequencing read length of single-ended 1X50 bp.

\section{qRT-PCR Validation of candidate miRNAs}

The expression of differentially expressed miRNAs in the sequencing results was validated by qRT-PCR. The template complementary DNA (CDNA) was synthesized using the PrimeScript RT kit (China Dalian, Takara), with slightly modified random primers, as per the manufacturer's instructions. Then, we used the resulting CDNA as a template. RT- PCR amplification was conducted by SuperReal PreMix Plus (SYBR Green; TianGen BioTech, China) and analyzed using Prism 7500 SDS (Applied Biosystems, Thermo Fisher Scientific, USA). The amplification steps included heating to $95^{\circ} \mathrm{C}$ for 10 minutes, $95^{\circ} \mathrm{C}$ for 40 cycles of 10 seconds each, and then $60^{\circ} \mathrm{C}$ for 15 seconds. The last step was to cool down to $72{ }^{\circ} \mathrm{C}$ for 20 seconds. GAPDH was used as an internal control for measuring RNA expression. The relative miRNA expression was calculated using $2^{-\Delta \Delta C t}$ method.

\section{miRNAs-gene-GO and miRNA-gene-KEGG interaction networks}

Several studies have indicated that miRNAs act as negative regulatory factors for regulating various physiological processes within the body[23, 24]. In order to explore the possible function of microRNAs from PDEs in coronary artery ISR, the interaction of miRNA-gene-GO and miRNA-gene-KEGG was predicted based on the in silico prediction methods TargetScan and Miranda. Firstly,gene Ontology (GO) functional significance enrichment analysis mapped all significantly differentially expressed genes to each term within the GO database, and the number of genes was calculated within each term. We then used hypergeometric test to identify the $\mathrm{GO}$ items that were enriched in significant differentially expressed genes compared to the whole genome background.

Kyoto Encyclopedia of Genes and Genomes (KEGG) not only provides all possible metabolic pathways, but also comprehensively annotates each enzymes that catalyzes each step of the reaction, including amino acid sequence, prorein data bank library, and other characteristics. Therefore we built miRNA-gene-GO network and miRNA-gene-KEGG network diagram using the Cytoscape 3.7.2 software.

\section{Statistical analysis}

All data is expressed as an average \pm standard error of the mean(SEM). Non-paired t-test was utilized for comparison between the restenosis and the non-restenosis group. $\mathrm{P}<0.05$ was considered statistically significant. All statistical analyses were conducted using GraphPad Prism 5.0 (GraphPad Prism Software Inc, San Diego, CA,USA) and SPSS software for Windows (version 25.0, SPSS Inc., Chicago, Illinois, USA).

Authors declare to confirm that all methods had been performed in accordance with relevant guidelines and regulations.

\section{Results}

\section{Clinical characteristic of patients}

We found no significant differences between groups with respect to age, gender, lipid profile,renal and liver function, glucose,or platelet counts ( Table 1). Three ISR patients and three controls were randomly selected for gene sequence (Table 2), and the remaining specimens were used by qRT-PCR for validation.

\section{Identification of exosomes}


After exosomes were extracted from patients' platelets, the expression of exosome markers CD9, CD69 and TSG101 were detected using western blotting (Fig. 1A). The exosomes revealed a typical cup shape under a transmission electron microscope, and with particle sizes of the exosome being approximately 100nm (Fig. 1B, C). These results indicated that we were able to successfully isolate platelet-derived granules, that were identified as exosomes.

\section{Genetic sequencing results}

We randomly selected three patients with restenosis and three patients without restenosis to undergo genetic sequencing. At first, we analyzed the small RNAs of PDEs by RNA sequencing. The results indicated that one miRNA was significantly upregulated while four miRNAs were down-regulated in the in-stent restenosis group $(P<0.05)$. The identified miRNAs for control vs ISR groups are outlined in Venn diagrams (Fig.2A). A volcano map and graded clustering heat map indicated significant different regards to differentiation and aggregation of miRNA expression between the restenosis and non-restenosis group (Fig. 2B, C). One of the five differentially expressed miRNAs is previously unknown with a sequence of $5^{\prime}$ CAGGCCCTGACGGATACGG 3'. We have named this miRNA PC-3p-75179_24. The remain four miRNAs have been previously identified according to microRNAs databases.They are hsa-miR-133a-3p_R+1, hsa-miR-548ap-5p_R+3, hsa-miR-589-5p_R-1 and hsa-miR-655-3p. And the differentially expressed miRNAs are shown in Table 3.

\section{QRT-PCR-validated differential expression of miRNAs in 20 patients}

In order to validate the difference in expression of miRNAs,PC-3p-75179_24, hsa-miR-133a-3p_R+1, hsa-miR-548ap-5p_R+3, hsa-miR-589-5p_R-1 and hsa-miR-655-3p, we carried out qRT-PCR. We chose 20 patients, including 10 patients with restenosis and 10 patients without restenosis. The results indicated that the expression of PC-3p-75179_24 was up-regulated while the expression of hsa-miR-133a-3p_R+1 and hsa-miR-589-5p_R-1 was down-regulated $(P<0.05)$ (Fig. 3). These results were consistent with prior RNA sequencing results. However, there were no significant differences in the expression of hsa-miR548ap-5p_R+3 and hsa-miR-655-3p, which may need to be further verified using a larger sample size.

\section{MiRNA-gene-GO network and MiRNA-gene-KEGG network diagram}

The target genes of each of the miRNAs were predicted using TargetScan and miRanda. The target base scores of each of the two software were predicted and selected as per the scoring standard of each software. Based on results from both TargetScan and miRanda, we constructed the miRNA-gene-GO interaction network diagrams of PC-3p-75179_24, hsa-miR133a-3p_R+1 and hsa-miR-589-5p_R-1. For each miRNA, we found that several genes were able to regulate each microRNA (Fig.4A). We continue to identify results from enrichment analysis of the top 25 target genes that are related to cardiovascular disease, and selected as part of the KEGG-pathway that we focus on as nodes. We also sorted out the miRNA and target genes that were related to this pathway in order to draw the network diagram with Cytoscape 3.7.2 software. The potential pathways of these three miRNAs are outlined in Fig. 4B.

\section{Discussion}

Thus far, approximately 2200 miRNAs have been identified within the mammalian genome, of which more than 1000 belong to the human genome[25]. In this study, we identified that the the newly discovered miRNA PC-3p-75179_24 was significantly up-regulated while hsa-miR-133a-3p_R + 1 and hsa-miR-589-5p_R-1 were significantly down-regulated within the restenosis group. Additionally we further verified this observation using the blood of select clinical patients, which showed that the results were consistent. Prior studies have shown that the miR-133a-3p, which is down-regulated in atherosclerosis, inhibits the proliferation, but induces apoptosis of human vascular smooth muscle cell (hVSMC) in MMP-9 dependent mechanisms[26]. In the BV2 cells treated with lipopolysaccharide (LPS) or chylomicrons (CM) after ischemia and reperfusion, miRNA-589 expression was found to be down-regulated, while overexpression of miRNA-589 decreased the release of inflammatory cytokines by targeting TRAF6[27]. It has been shown that histone deacetylase (HDACs) expression is negatively correlated with expression of the tumor suppressor miR-589-5p in NSCLC samples. Specifically, phenotypic experiments in vitro and in vivo shown that miR-589-5p/HDAC5 pathway has a vital function in migration, invasion and tumorigenicity of 
NSCLC cells[28]. Furthermore, miRNA-589 is a tumor suppressor that directly targets breast cancer metastasis-associated protein[29]. MiRNA PC-3p-75179_24 has never been reported. The role of this miRNA in ISR may be an interesting and important topic.

Exosomes can founction a bridge for transportation of functional miRNAs to their target cells. In addition, as a medium of cellular communication, exosomes may be transported through the endothelial monolayer and infiltrate into VSMCs. Zeng Wang et al. constructed a co-culture model of endothelial monolayer and VSMCs in order to study the osmotic effect of exosomes that were derived from macrophages. The exosome was absorbed by human aortic vascular smooth muscle cells (HA-VSMCs) $12 \mathrm{~h}$ after addition, and the fluorescence intensity of HA-VSMCs within the cytoplasm increased with time. Hence,exosomes have the ability to enter smooth muscle cells across endothelial cells[30].

We found three miRNAs from PDEs that were differentially expressed in patients with ISR. Hence, we constructed a miRNAsgene-GO and miRNAs-gene-KEGG network diagram of the three different miRNAs in order to identify their potential roles. As shown in Fig. 4, among the these specific genes and pathways, previous studies showed that MMP-14, ICAM-1, TLR4, and Ras signaling pathway, AMPK signaling pathway play important roles in the development and progression of atherosclerosis, (i.e. proliferation and migration of vascular smooth cells), while modulating vascular remodeling and inflammation[31-35]. Hence, the miRNA-gene-GO network and miRNA-gene-KEGG network diagram provide novel avenue for searching for potential diagnostic biomarkers, and also provide a novel direction for assessing the mechanism of these miRNAs in ISR. Further investigation is needed to evaluate whether these miRNAs have a function in the development of ISR.

\section{Conclusion}

In summary, our study provides the first data regarding differential expression of miRNAs in PDEs in plasma from patients with ISR. MiRNA PC-3p-75179_24, hsa-miR-133a-3p_R + 1 and hsa-miR-589-5p_R-1 had significantly differential expression. Further studies are needed to assess the potential of these miRNAs as biomarkers and pathologic mediators of ISR.

\section{Abbreviations}

ISR:In-stent restenosis

PCl:Percutaneous coronary intervention

PDEs:Platelet-derived Exosomes

VSMC:vascular smooth muscle cell

NTA:Nanoparticle Tracking Analysis

TEM:transmission electron microscopy

GO:Gene Ontology

KEGG:Kyoto Encyclopedia of Genes and Genomes

LPS:lipopolysaccharide

HDAC:histone deacetylase

NSCLC:non-small cell lung cancer

$\mathrm{CM}$ :chylomicrons

BP:biological process 
CC:cell component

MF:molecular function

\section{Declarations}

\section{Ethics approval and consent to participate}

All participants provided written consent before entering the study, and approval was obtained from the Human Subjects Committee at the Zhongda Hospital, Southeast University.

\section{Consent for publication}

Not applicable.

\section{Availability of data and materials}

The datasets used analysed during the current study are available from the corresponding author on reasonable request.

\section{Competing interests}

The authors report no conflicts of interest.

\section{Funding}

This study was supported by the National Science Foundation of China (Research Grant No.81970237). The funders had no role in study design, data collection and analysis, preparation of the manuscript or the decision to submit the article for publication.

\section{Authors' contributions}

HLD conceived, designed the study and drafted the manuscript. QY, LLQ recruited the participants and collected the data. WD,QYH and YGL analysed and interpreted the data. TCC critically revised the manuscript. All authors read and approved the final manuscript.

\section{Acknowledgements}

The authors thanks Professor Yao Yuyu for her guidance in scientific research ideas and research direction. We thank all authors for reviewing, statistical analyses and all the participants for their contributions.

\section{References}

1. Shishido K, Antoniadis AP, Takahashi S, Tsuda M, Mizuno S, Andreou I, Papafaklis MI, Coskun AU, O'Brien C, Feldman CL et al: Effects of Low Endothelial Shear Stress After Stent Implantation on Subsequent Neointimal Hyperplasia and Clinical Outcomes in Humans. J AM HEART ASSOC 2016, 5(9).

2. Dangas GD, Claessen BE, Caixeta A, Sanidas EA, Mintz GS, Mehran R: In-stent restenosis in the drug-eluting stent era. J AM COLL CARDIOL 2010, 56(23):1897-1907.

3. Oba Y, Ogata N, Fukutomi M, Kario K: Usefulness of three-dimensional optical frequency domain imaging for diagnosing in-stent restenosis due to a stent fracture. EUROINTERVENTION 2016, 12(11):e1438.

4. Ishibashi M, Hiasa K, Zhao Q, Inoue S, Ohtani K, Kitamoto S, Tsuchihashi M, Sugaya T, Charo IF, Kura S et al: Critical role of monocyte chemoattractant protein-1 receptor CCR2 on monocytes in hypertension-induced vascular inflammation and remodeling. CIRC RES 2004, 94(9):1203-1210. 
5. Charo IF, Ransohoff RM: The many roles of chemokines and chemokine receptors in inflammation. N Engl J Med 2006, 354(6):610-621.

6. Edfeldt K, Swedenborg J, Hansson GK, Yan ZQ: Expression of toll-like receptors in human atherosclerotic lesions: a possible pathway for plaque activation. CIRCULATION 2002, 105(10):1158-1161.

7. Lee MS, David EM, Makkar RR, Wilentz JR: Molecular and cellular basis of restenosis after percutaneous coronary intervention: the intertwining roles of platelets, leukocytes, and the coagulation-fibrinolysis system. J PATHOL 2004, 203(4):861-870.

8. Raposo G, Stoorvogel W: Extracellular vesicles: exosomes, microvesicles, and friends. J CELL BIOL 2013, 200(4):373-383.

9. Ludwig AK, Giebel B: Exosomes: small vesicles participating in intercellular communication. Int J Biochem Cell Biol 2012, 44(1):11-15.

10. Kalra H, Simpson RJ, Ji H, Aikawa E, Altevogt P, Askenase P, Bond VC, Borras FE, Breakefield X, Budnik V et al: Vesiclepedia: a compendium for extracellular vesicles with continuous community annotation. PLOS BIOL 2012, 10(12):e1001450.

11. Conde-Vancells J, Rodriguez-Suarez E, Embade N, Gil D, Matthiesen R, Valle M, Elortza F, Lu SC, Mato JM, Falcon-Perez JM: Characterization and comprehensive proteome profiling of exosomes secreted by hepatocytes. J PROTEOME RES 2008, 7(12):5157-5166.

12. Mathivanan S, Fahner CJ, Reid GE, Simpson RJ: ExoCarta 2012: database of exosomal proteins, RNA and lipids. NUCLEIC ACIDS RES 2012, 40(Database issue):D1241-D1244.

13. Bei Y, Das S, Rodosthenous RS, Holvoet P, Vanhaverbeke M, Monteiro MC, Monteiro V, Radosinska J, Bartekova M, Jansen F et al: Extracellular Vesicles in Cardiovascular Theranostics. THERANOSTICS 2017, 7(17):4168-4182.

14. Iorio MV, Croce CM: MicroRNA dysregulation in cancer: diagnostics, monitoring and therapeutics. A comprehensive review. EMBO MOL MED 2017, 9(6):852.

15. Pasquinelli AE: MicroRNAs and their targets: recognition, regulation and an emerging reciprocal relationship. NAT REV GENET 2012, 13(4):271-282.

16. Bartel DP: MicroRNAs: genomics, biogenesis, mechanism, and function. CELL 2004, 116(2):281-297.

17. Cui RR, Li SJ, Liu LJ, Yi L, Liang QH, Zhu X, Liu GY, Liu Y, Wu SS, Liao XB et al: MicroRNA-204 regulates vascular smooth muscle cell calcification in vitro and in vivo. CARDIOVASC RES 2012, 96(2):320-329.

18. Liao XB, Zhang ZY, Yuan K, Liu Y, Feng X, Cui RR, Hu YR, Yuan ZS, Gu L, Li SJ et al: MiR-133a modulates osteogenic differentiation of vascular smooth muscle cells. ENDOCRINOLOGY 2013, 154(9):3344-3352.

19. Tan M, Yan HB, Li JN, Li WK, Fu YY, Chen W, Zhou Z: Thrombin Stimulated Platelet-Derived Exosomes Inhibit PlateletDerived Growth Factor Receptor-Beta Expression in Vascular Smooth Muscle Cells. CELL PHYSIOL BIOCHEM 2016, 38(6):2348-2365.

20. Li J, Tan M, Xiang Q, Zhou Z, Yan H: Thrombin-activated platelet-derived exosomes regulate endothelial cell expression of ICAM-1 via microRNA-223 during the thrombosis-inflammation response. THROMB RES 2017, 154:96-105.

21. Gidlof O, van der Brug M, Ohman J, Gilje P, Olde B, Wahlestedt C, Erlinge D: Platelets activated during myocardial infarction release functional miRNA, which can be taken up by endothelial cells and regulate ICAM1 expression. BLOOD 2013, 121(19):3908-3917, S1-S26.

22. Chistiakov DA, Orekhov AN, Bobryshev YV: Extracellular vesicles and atherosclerotic disease. CELL MOL LIFE SCI 2015, 72(14):2697-2708.

23. Szuk T, Fejes Z, Debreceni IB, Kerenyi A, Edes I, Kappelmayer J, Nagy BJ: Integrity((R)) bare-metal coronary stent-induced platelet and endothelial cell activation results in a higher risk of restenosis compared to Xience((R)) everolimus-eluting stents in stable angina patients. PLATELETS 2016, 27(5):410-419.

24. Vanags LZ, Tan J, Galougahi KK, Schaefer A, Wise SG, Murphy A, Ali ZA, Bursill CA: Apolipoprotein A-I Reduces In-Stent Restenosis and Platelet Activation and Alters Neointimal Cellular Phenotype. JACC Basic Transl Sci 2018, 3(2):200-209.

25. Ardekani AM, Naeini MM: The Role of MicroRNAs in Human Diseases. Avicenna J Med Biotechnol 2010, 2(4):161-179. 
26. Shi L, Yu C, Tian X, Ma C, Wang L, Xia D, Cui C, Chen X, Jiang T, Gu Y et al: Effect of microRNA-133a-3p/matrix metalloproteinase-9 axis on the growth of atherosclerotic vascular smooth muscle cells. EXP THER MED 2019, 18(6):4356-4362.

27. Ma GP, Yang BZ, Zhang YS, Wang B, Wei XH, Zhang RF, Jia KH, Gao JP: Protective effects of miRNA-589 on cerebral ischemia-reperfusion injury. J Biol Regul Homeost Agents 2020, 34(4):1269-1275.

28. Liu C, Lv D, Li M, Zhang X, Sun G, Bai Y, Chang D: Hypermethylation of miRNA-589 promoter leads to upregulation of HDAC5 which promotes malignancy in non-small cell lung cancer. INT J ONCOL 2017, 50(6):2079-2090.

29. Chu J: MicroRNA-589 serves as a tumor suppressor microRNA through directly targeting metastasis-associated protein 2 in breast cancer. ONCOL LETT 2019, 18(3):2232-2239.

30. Ma GP, Yang BZ, Zhang YS, Wang B, Wei XH, Zhang RF, Jia KH, Gao JP: Protective effects of miRNA-589 on cerebral ischemia-reperfusion injury. J Biol Regul Homeost Agents 2020, 34(4):1269-1275.

31. Park JH, Park SM, Park SH, Cho KH, Lee ST: Cleavage and functional loss of human apolipoprotein E by digestion of matrix metalloproteinase-14. PROTEOMICS 2008, 8(14):2926-2935.

32. Tishko VV, Sokolov AA, Belskih AN, Ivanov AM, Meshkova ME, Skorinova TS: Impact of double filtration plasmapheresis on adhesion molecules levels in patients with stable coronary heart disease after coronary stenting. Atheroscler Suppl 2017, 30:92-98.

33. Roshan MH, Tambo A, Pace NP: The Role of TLR2, TLR4, and TLR9 in the Pathogenesis of Atherosclerosis. Int J Inflam 2016, 2016:1532832.

34. Yu MH, Lin MC, Huang CN, Chan KC, Wang CJ: Acarbose inhibits the proliferation and migration of vascular smooth muscle cells via targeting Ras signaling. Vascul Pharmacol 2018, 103-105:8-15.

35. Peng LP, Cao Y, Zhao SL, Huang YX, Yang K, Huang W: Memory T cells delay the progression of atherosclerosis via AMPK signaling pathway. Ann Transl Med 2019, 7(23):782.

\section{Tables}

Table 1

Clinical characteristics of total patients

\begin{tabular}{|lllll|}
\hline & All patients & ISR( $\mathbf{n}=13)$ & Control $(\mathbf{n}=13)$ & P value \\
\hline Age, y & $64.81 \pm 6.39$ & $64.31 \pm 6.72$ & $65.31 \pm 6.22$ & 0.697 \\
\hline Male gender & $61.54 \%$ & $61.54 \%$ & $61.54 \%$ & 0.14 \\
\hline Glucose(mmol/L) & $6.2 \pm 1.30$ & $6.14 \pm 1.45$ & $6.34 \pm 1.18$ & 0.698 \\
\hline Cr(mmol/L) & $81.57 \pm 43.59$ & $75.23 \pm 27.21$ & $87.92 \pm 55.94$ & 0.469 \\
\hline ALT(IU/L) & $28.08 \pm 11.76$ & $29.92 \pm 13.29$ & $26.23 \pm 10.21$ & 0.435 \\
\hline $\operatorname{LDL}-\mathrm{C}(\mathrm{mmol} / \mathrm{L})$ & $2.25 \pm 0.15$ & $2.28 \pm 0.63$ & $2.21 \pm 0.87$ & 0.799 \\
\hline $\mathrm{PLT}\left(\times 10^{9} / \mathrm{L}\right)$ & $193.48 \pm 46.67$ & $194.00 \pm 47.71$ & $192.92 \pm 47.63$ & 0.955 \\
\hline
\end{tabular}


Table 2

Clinical characteristics of 6 patients.

\begin{tabular}{|llll|}
\hline Patient number & Age,y & Gender & ISR or control \\
\hline Case1 & 68 & Female & ISR1 \\
\hline Case2 & 62 & Male & ISR2 \\
\hline Case3 & 59 & Male & ISR3 \\
\hline Case4 & 71 & Male & Control1 \\
\hline Case5 & 58 & Male & Control2 \\
\hline Case6 & 62 & Female & Control3 \\
\hline
\end{tabular}

Table 3

The differentially expressed miRNAs

\begin{tabular}{|c|c|c|c|c|c|c|}
\hline Index & $\begin{array}{l}\text { miRNA } \\
\text { name }\end{array}$ & $\begin{array}{l}\text { miRNA sequence } \\
\left(5^{\prime} \text { to } 3^{\prime}\right)\end{array}$ & up/down & fold_change & log2(fold_change) & pvalue(t_test) \\
\hline 1 & $\begin{array}{l}\text { PC-3p- } \\
\text { 75179_24 }\end{array}$ & CAGGCCCTGACGGATACGG & up & 19.39 & 4.28 & $5.76 \mathrm{E}-04$ \\
\hline 2 & $\begin{array}{l}\text { hsa-miR- } \\
\text { 133a- } \\
3 p \_R+1\end{array}$ & TTTGGTCCCCTTCAACCAGCTGT & down & 0.23 & -2.14 & 2.16E-02 \\
\hline 3 & $\begin{array}{l}\text { hsa-miR- } \\
\text { 589-5p_R- } \\
1\end{array}$ & AAAAGTAATTGCGGTCTTTGGT & down & 0.18 & -2.44 & 2.69E-02 \\
\hline 4 & $\begin{array}{l}\text { hsa-miR- } \\
\text { 589-5p_R- } \\
1\end{array}$ & TGAGAACCACGTCTGCTCTGA & down & 0.21 & -2.27 & 4.44E-02 \\
\hline 5 & $\begin{array}{l}\text { hsa-miR- } \\
655-3 p\end{array}$ & ATAATACATGGTTAACCTCTTT & down & 0.09 & -3.49 & 4.97E-02 \\
\hline
\end{tabular}

\section{Figures}


$(A)$

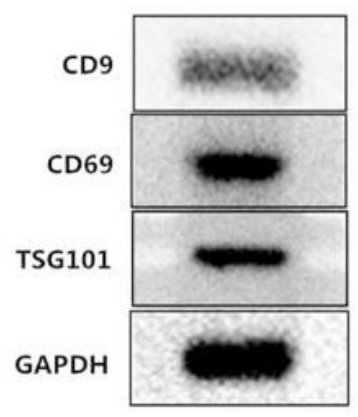

(B)

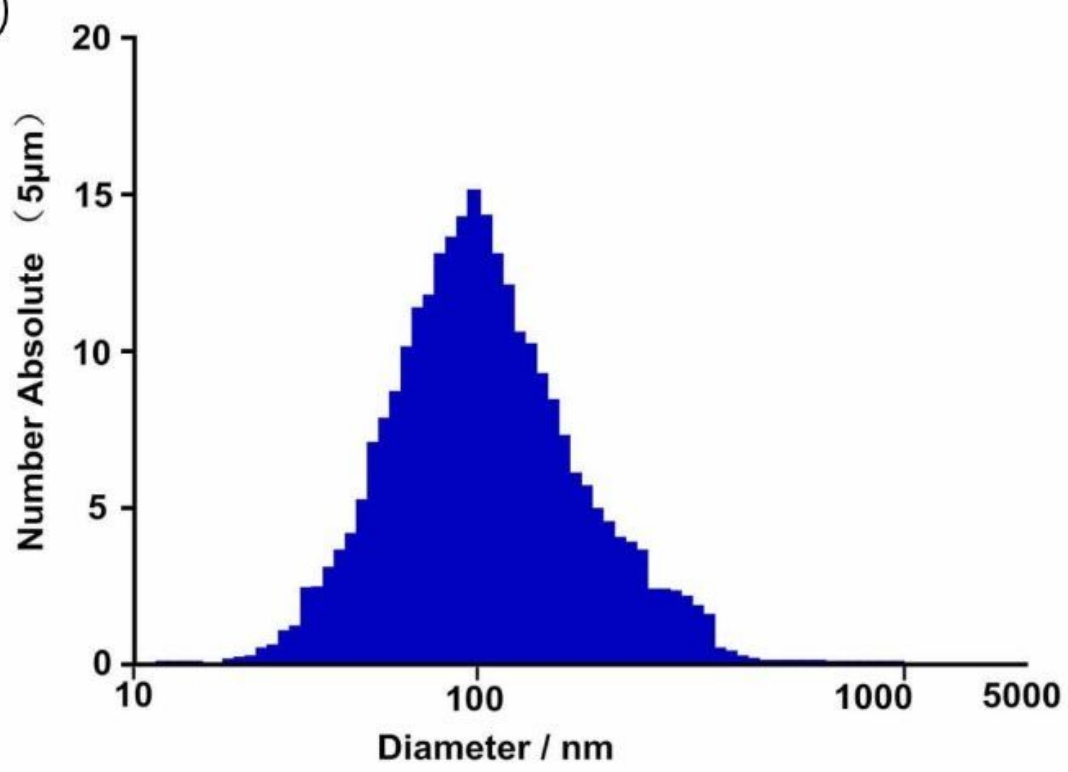

(C)

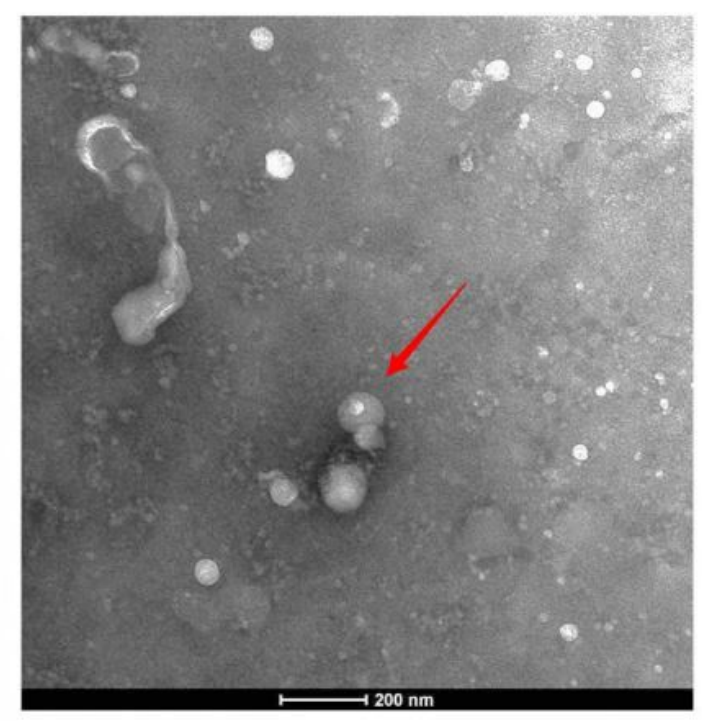

\section{Exosome derived from platelet}

\section{Figure 1}

Characterization of platelet-derived exosomes. (A) PDEs were validated by of CD9, CD69, TSG101 and GAPDH using western blotting. (B) NTA measurements size of plasma exosomes. (C) Visualized of PDEs by TEM. NTA( Nanoparticle Tracking Analysis); TEM (transmission electron microscopy). 
Venn diagrams of detected miRNAs(control vs ISR)

(A)

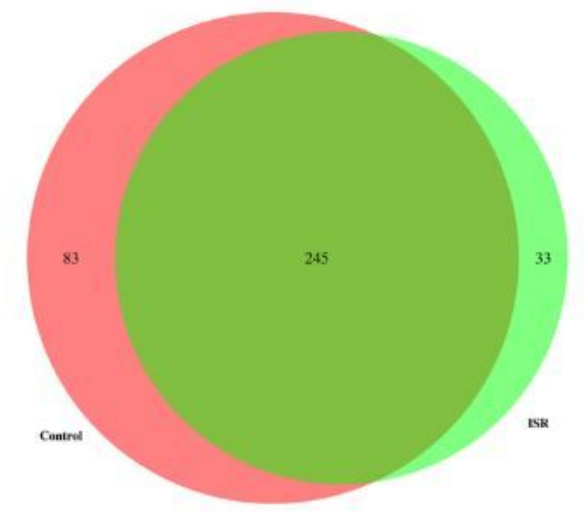

(B)

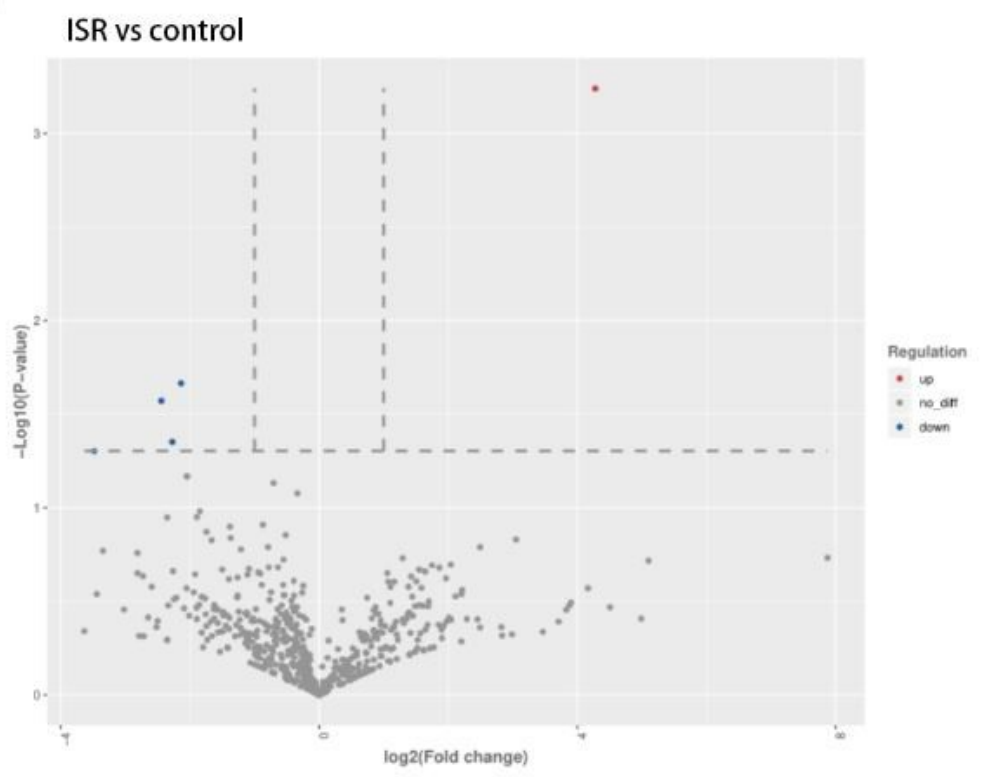

(C)

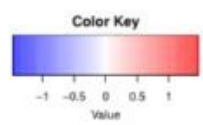

PC-3p-75179_24

hsa-miR-133a-3p_R+1

hsa-miR-548ap-5p_R+3

hsa-miR-589-5p_R-1

hsa-miR-655-3p

\section{Figure 2}

Analysis of differentially expressed microRNAs,determined by RNA sequencing, in plasma exosomes from ISR patients compared to controls. (A) Venn diagrams of miRNAs for control vs ISR patients. (B) Scatter plots are utilized to assess changes in microRNAs expression between ISR patients and the control group. Taking the line $(P<0.05)$ as the boundary, blue points are on behave of the down-regulated microRNAs, the red point is on behave of the up-regulated microRNA. (C) Hierarchical clustering presents a distinct expression profile of microRNAs between ISR patients and the control group $(\mathrm{P}<0.05)$. ISR (in-stent restenosis) 
(A)

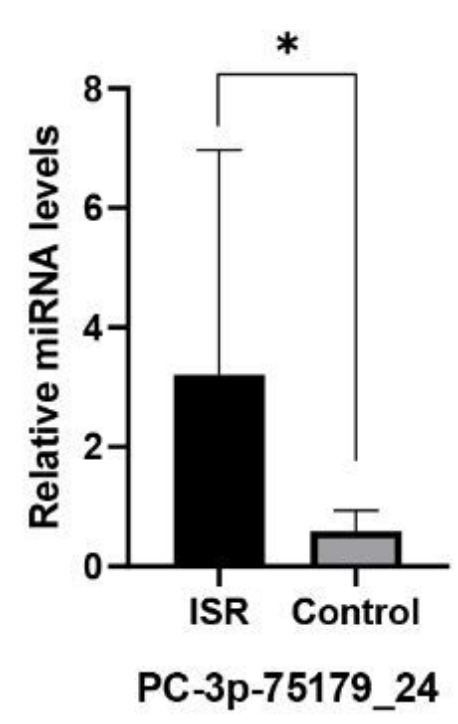

(B)

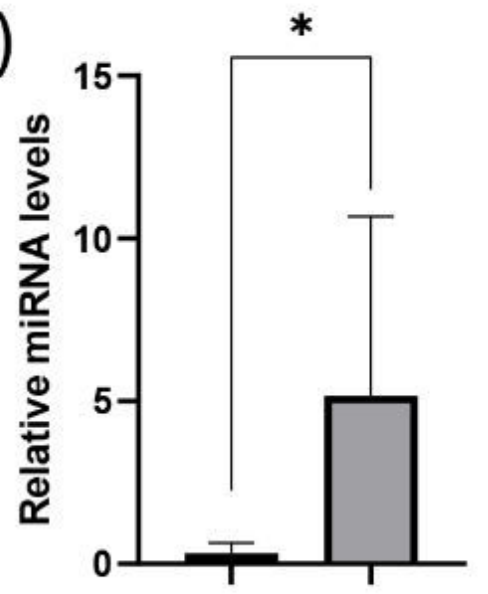

hsa-miR-133a-3p_R+1
(C)

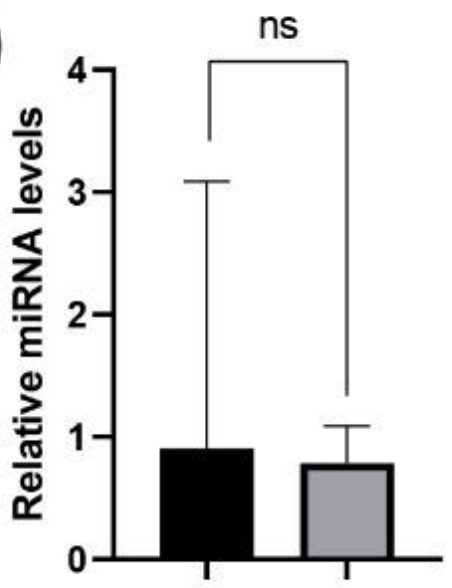

hsa-miR-548ap-5p_R+3
(D)

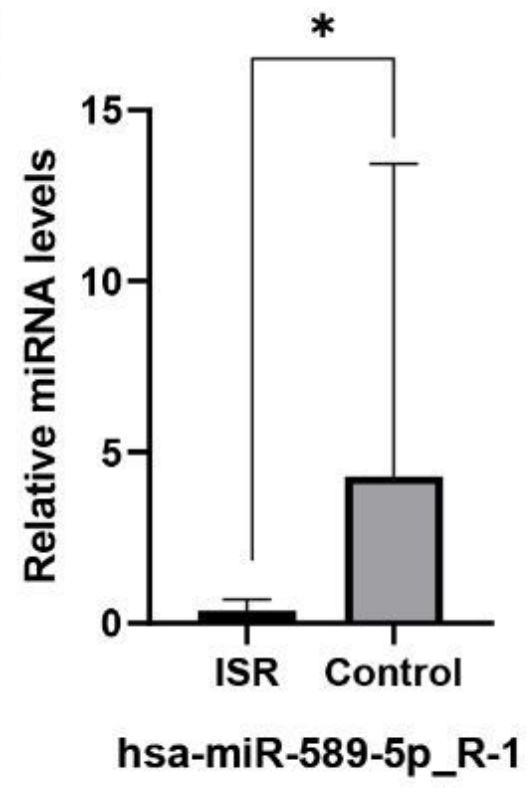

(E)

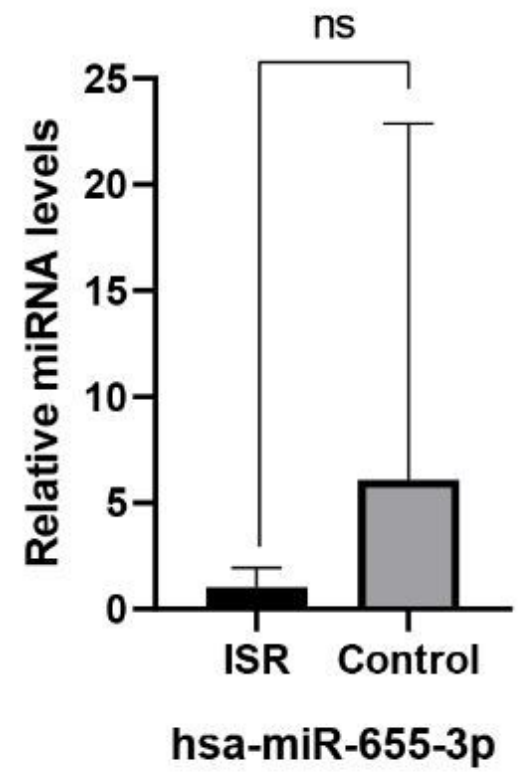

Figure 3

Validation of miRNAs expression by qRT-PCR. All values are expressed as average value \pm S.E.M. (A)Level of miRNA PC-3p75179_24 compared to the control group $\left(n=10 ;{ }^{*} p<0\right.$. 05). (B)Level of miRNA hsa-miR-133a-3p_R+1 compared to the control group $\left(n=10,{ }^{*} p<0.05\right)$. (C) Level of miRNA hsa-miR-548ap-5p_R+3 compared to the control group $(n=10$, * $p>0$. 05). (D) Level of hsa-miR-589-5p_R-1 compared to the control group $\left(n=10 ;{ }^{\star} p<0.05\right)$. (E) Level of hsa-miR-655-3p compared to the control group $\left(n=10,{ }^{\star} p>0.05\right)$. 
(A)

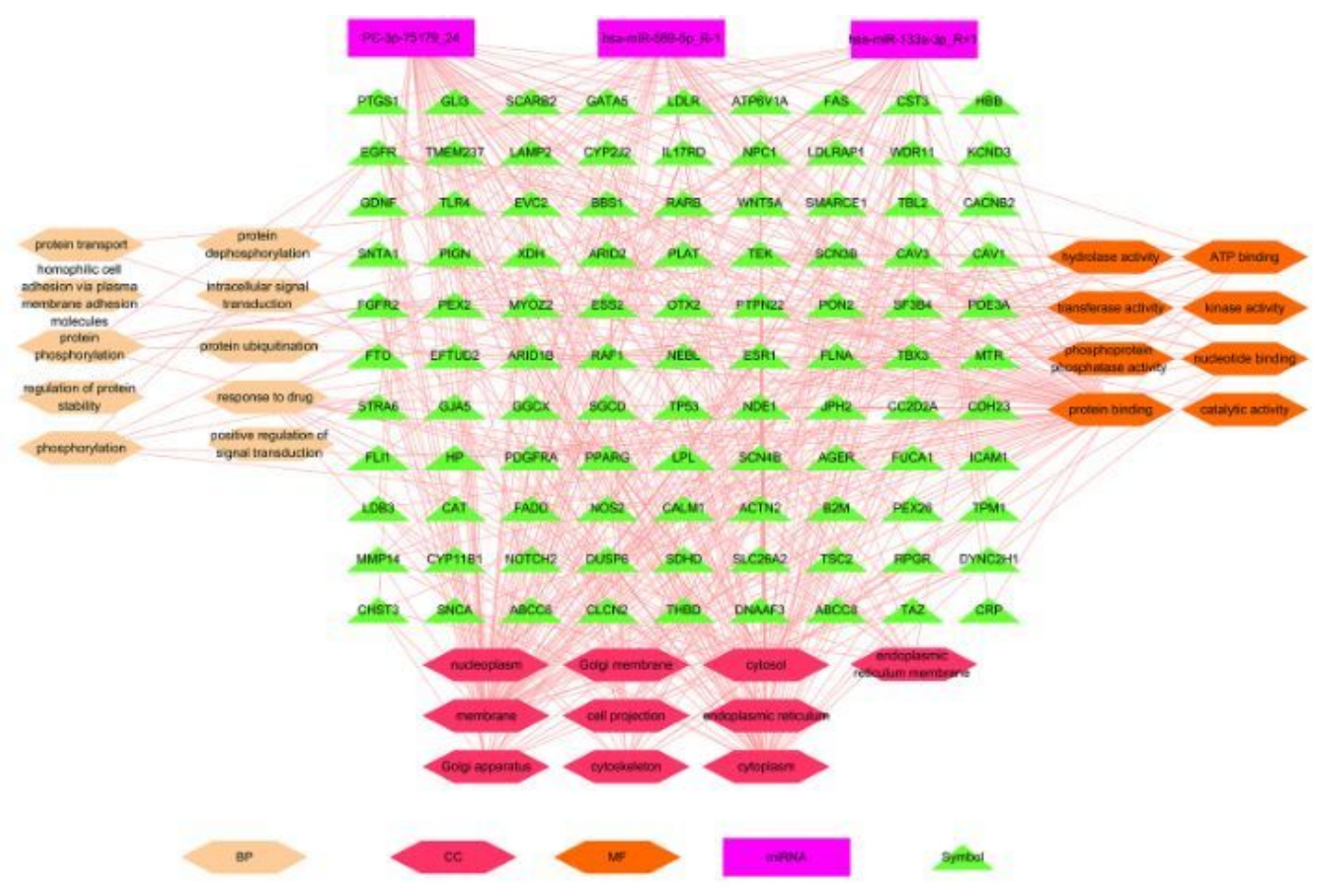

(B)

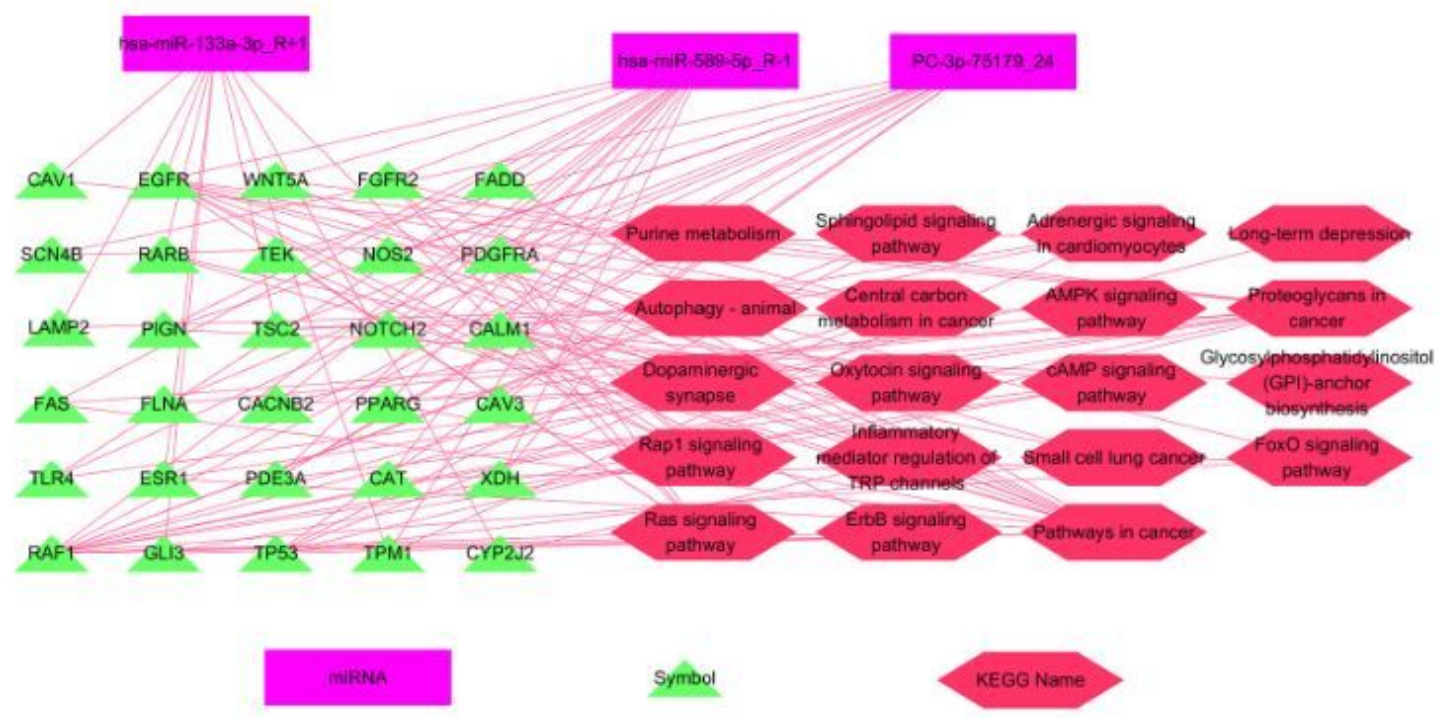

Figure 4

MiRNA-gene-GO and miRNA-gene-KEGG interactions analysis for PC-3p-75179_24, hsa-miR-133a-3p_R+1 and hsa-miR-5895p_R-1 based on in silico prediction and bioinformatics analysis. (A) The enrichment analysis of target genes, The GO term of interest was selected as the node. The BP, CC, MF related to this $\mathrm{GO}$ term was obtained and network diagram was drawn with Cytoscape 3.7.2 software. (B) The possible pathways and the top 25 target genes that were related to cardiovascular disease and may be regulated by these miRNAs. BP( biological process); CC (cell component); MF (molecular function).

\section{Supplementary Files}

This is a list of supplementary files associated with this preprint. Click to download.

- CD69.tif

- CD9.tif

- GAPDH.tif 
- TSG1.tif

Page 15/15 